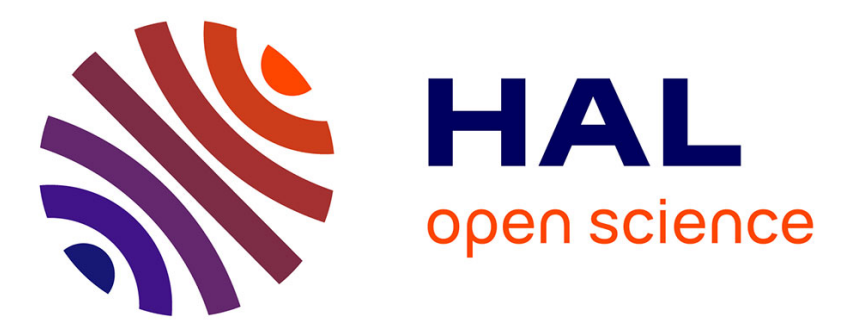

\title{
Oxidative stress regulates autophagy in cultured muscle cells of patients with chronic obstructive pulmonary disease
}

Fares Gouzi, Marine Blaquière, Matthias Catteau, François Bughin, Jonathan

Maury, Emilie Passerieux, Bronia Ayoub, Jacques Mercier, Maurice Hayot, Pascal Pomiès

\section{To cite this version:}

Fares Gouzi, Marine Blaquière, Matthias Catteau, François Bughin, Jonathan Maury, et al.. Oxidative stress regulates autophagy in cultured muscle cells of patients with chronic obstructive pulmonary disease. Journal of Cellular Physiology, 2018, 10.1002/jcp.26868 . hal-01824722

\section{HAL Id: hal-01824722 \\ https://hal.umontpellier.fr/hal-01824722}

Submitted on 25 Nov 2019

HAL is a multi-disciplinary open access archive for the deposit and dissemination of scientific research documents, whether they are published or not. The documents may come from teaching and research institutions in France or abroad, or from public or private research centers.
L'archive ouverte pluridisciplinaire HAL, est destinée au dépôt et à la diffusion de documents scientifiques de niveau recherche, publiés ou non, émanant des établissements d'enseignement et de recherche français ou étrangers, des laboratoires publics ou privés. 


\title{
Oxidative stress regulates autophagy in cultured muscle cells of patients with chronic obstructive pulmonary disease
}

\author{
Fares Gouzi $^{1,2}$ | Marine Blaquière ${ }^{1,2}$ | Matthias Catteau ${ }^{1}$ | François Bughin ${ }^{1,2}$ | \\ Jonathan Maury ${ }^{1,3}$ | Emilie Passerieux ${ }^{1}$ | Bronia Ayoub ${ }^{1,2}$ | Jacques Mercier ${ }^{1,2}$ | \\ Maurice Hayot $^{1,2 *}$ | Pascal Pomiès ${ }^{1 *}$ (l)
}

${ }^{1}$ PhyMedExp, University of Montpellier, INSERM, CNRS, Montpellier, France

${ }^{2}$ Department of Clinical Physiology, CHRU of Montpellier, Montpellier, France

${ }^{3}$ Clinique du Souffle "La Solane,"

Fontalvie/5-Santé Group, Osséja, France

\section{Correspondence}

Pascal Pomiès, PhyMedExp, 371 Avenue du Doyen Giraud, CHRU Arnaud de Villeneuve, 34295 Montpellier Cedex 5, France.

Email: pascal.pomies@inserm.fr

Funding information

CHRU of Montpellier, PHRC (AREB-1);

Apard (Association Pour l'Assistance

et la Réhabilitation à Domicile)
The proteolytic autophagy pathway is enhanced in the lower limb muscles of patients with chronic obstructive pulmonary disease (COPD). Reactive oxygen species (ROS) have been shown to regulate autophagy in the skeletal muscles, but the role of oxidative stress in the muscle autophagy of patients with COPD is unknown. We used cultured myoblasts and myotubes from the quadriceps of eight healthy subjects and twelve patients with COPD (FEV1\% predicted: $102.0 \%$ and $32.0 \%$, respectively; $p$ <0.0001). We compared the autophagosome formation, the expression of autophagy markers, and the autophagic flux in healthy subjects and the patients with COPD, and we evaluated the effects of the 3-methyladenine (3-MA) autophagy inhibitor on the atrophy of COPD myotubes. Autophagy was also assessed in COPD myotubes treated with an antioxidant molecule, ascorbic acid. Autophagosome formation was increased in COPD myoblasts and myotubes ( $p=0.011 ; p<0.001)$, and the LC3 2/LC3 1 ratio $(p=0.002)$, SQSTM1 mRNA and protein expression $(p=0.023$; $p=0.007)$, BNIP3 expression ( $p=0.031)$, and autophagic flux $(p=0.002)$ were higher in COPD myoblasts. Inhibition of autophagy with 3-MA increased the COPD myotube diameter $(p<0.001)$ to a level similar to the diameter of healthy subject myotubes. Treatment of COPD myotubes with ascorbic acid decreased ROS concentration $(p<0.001)$, ROS-induced protein carbonylation $(p=0.019)$, the LC3 2/LC3 1 ratio $(p=0.037)$, the expression of SQSTM1 $(p<0.001)$ and BNIP3 $(p<0.001)$, and increased the COPD myotube diameter $(p<0.001)$. Thus, autophagy signaling is enhanced in cultured COPD muscle cells. Furthermore, the oxidative stress level contributes to the regulation of autophagy, which is involved in the atrophy of COPD myotubes in vitro.

\section{KEYWORDS}

atrophy, autophagy, chronic obstructive pulmonary disease (COPD), oxidative stress, satellite cells

\section{1 | INTRODUCTION}


with COPD, muscle dysfunction has a crucial impact on the quality of life and mortality rate of patients. Muscle dysfunction is associated with reduced muscle strength and endurance and is often accompanied by muscle atrophy of the lower limbs, which has been attributed in great part to increased protein degradation due to activation of the ubiquitin/proteasome pathway (Doucet et al., 2007; Plant et al., 2010).

The autophagy-lysosome pathway is a proteolytic system that contributes to muscle homeostasis through the elimination of protein aggregates and damaged organelles. Autophagy has been shown to be induced in skeletal muscles in response to various pro-atrophic stimuli such as starvation (Mizushima, Yamamoto, Matsui, Yoshimori, \& Ohsumi, 2004), denervation-induced muscle disuse (O'Leary, Vainshtein, Carter, Zhang, \& Hood, 2012), hypoxia (Bellot et al., 2009), and oxidative stress (Dobrowolny et al., 2008). Furthermore, use of the antioxidant molecule $\mathrm{N}$-acetylcysteine (NAC) has shown that reactive oxygen species (ROS) regulate autophagy in in vitro myogenic $\mathrm{C} 2 \mathrm{C} 12$ cells, as well as in the skeletal muscles of mice (Qi, He, Ji, \& Ding, 2014; Rahman et al., 2014). In COPD, autophagy signaling is enhanced in the tibialis anterior and vastus lateralis of stable patients (Guo et al., 2013; Puig-Vilanova et al., 2015). Moreover, indirect measurements suggest that oxidative stress might contribute to autophagy, and autophagy markers are inversely correlated with thigh atrophy and lung function impairment in patients with COPD (Guo et al., 2013). Nevertheless, while ROS have been shown to regulate autophagy in skeletal muscles, the involvement of oxidative stress in COPD muscle autophagy has yet to be clearly demonstrated.

We recently developed a cellular model based on purified satellite cells derived from the quadriceps of patients with COPD and showed that oxidative stress is involved in the atrophy of cultured COPD myotubes via the ubiquitin/proteasome signaling pathway (Pomiès et al., 2015, 2016). Here, we use this cellular model to assess the role of autophagy in the atrophy of COPD muscle cells in vitro and to investigate whether oxidative stress is involved in COPD muscle autophagy. Autophagy signaling was evaluated with various autophagy markers in in vitro muscle cells of patients with COPD and healthy subjects, while the effect of the autophagy inhibitor 3-methyladenine (3-MA) was tested on COPD myotube atrophy. Autophagy signaling was also assessed in cultured COPD myotubes subjected to antioxidant treatment.

\section{2 | MATERIALS AND METHODS}

\section{1 | Study population}

The two study groups, sedentary healthy subjects $(n=8)$ and patients with COPD ( $n=12)$, are identical to the groups we have previously studied (Pomiès et al., 2016), and their characteristics are detailed in Table 1. Anthropometry, pulmonary function, exercise level and peripheral muscle function were assessed, as previously described (Pomiès et al., 2016). All subjects gave an informed written consent, and the research protocol was approved by the institutional ethics
TABLE 1 Characteristics of the study groups

\begin{tabular}{|llll|}
\hline & Healthy subjects & COPD patients & P-value \\
\hline$n$ & 8 & 12 & - \\
\hline Gender (M/F) & $7 / 1$ & $9 / 3$ & - \\
\hline Age (year) & $62.0[57.0-67.5]$ & $59.0[54.0-62.8]$ & 0.393 \\
\hline BMI (kg/m²) & $25.9[24.3-27.8]$ & $21.6[19.0-26.0]$ & 0.071 \\
\hline FFMI (kg/m $\left.{ }^{2}\right)$ & $19.4[18.4-20.5]$ & $17.1[15.0-18.3]$ & 0.025 \\
\hline FEV1 (\% pred.) & $102.0[93.0-105.0]$ & $32.0[26.8-47.5]$ & $<0.0001$ \\
\hline FEV1/FVC (\%) & $75.0[68.8-81.5]$ & $39.0[32.0-41.0]$ & 0.002 \\
\hline 6MWD (\% pred.) & $88.0[83.5-97.0]$ & $68.0[55.5-71.5]$ & $<0.0001$ \\
\hline qMVC (kg) & $27.7[25.1-31.6]$ & $17.6[12.9-32.2]$ & 0.071 \\
\hline BODE index & - & $5.0[4.3-8.5]$ & - \\
\hline
\end{tabular}

Note. BMI, body mass index; COPD, chronic obstructive pulmonary disease; FFMI, fat-free mass index; FEV1, forced expiratory volume in $1 \mathrm{~s}$; FVC, forced vital capacity; 6MWD, 6-min walking distance; qMVC, quadriceps muscle voluntary contraction.

The BODE index takes into account the body mass index, the airflow obstruction, the functional dyspnea, and the exercise capacity (Celli et al., 2004). Values are presented as median [25th percentile-75th percentile].

committee of the Montpellier University Hospitals (no. 200904-BPCO-V2 and no. 2011-A00842-39) and conducted in accordance with the Helsinki Declaration and the European guidelines for "good clinical practice."

\section{2 | Muscle biopsy, myoblast purification, and cell culture}

Muscle biopsies were obtained from the vastus lateralis using our needle method (Gouzi et al., 2013). One piece of the biopsy was flash frozen in a liquid nitrogen precooled beaker of isopentane and then conserved at $-80^{\circ} \mathrm{C}$, until total RNA extraction using the NucleoSpin RNA/protein purification kit (Macherey-Nagel, Düren, Germany). Another piece of the biopsy was progressively frozen for later satellite cell purification, as described previously (Pomiès et al., 2015). Myoblast purification was performed using an immunomagnetic method, as previously described in detail (Pomiès et al., 2015).

The myoblasts were grown in collagen-coated petri dishes in proliferation medium: Dulbecco modified Eagle medium (DMEM)/20\% fetal bovine serum (FBS)/0.5\% Ultroser. Myoblasts were always cultured at a passage below P4. Differentiation was induced by replacing the proliferation medium with the differentiation medium: DMEM/2\% FBS. Myotubes were obtained after 5 days in differentiation medium.

\section{3 | Cell treatments}

Healthy and COPD myoblasts and myotubes were grown in the presence of $200 \mathrm{nM}$ bafilomycin A1 or phosphate-buffered saline (PBS) for $90 \mathrm{~min}$ before harvesting the cells. The LC3 2 autophagic flux was determined by calculating the difference between the LC3 2/tubulin ratio in the presence and in the absence of bafilomycin A1. 
TABLE 2 Primers used

\begin{tabular}{lllll} 
Gene & Forward primer & Reverse primer & & Product length \\
\hline SQSTM1 & GCACCTGTCTGAGGGCTTCT & GCTCCAGTTTCCTGGTGGAC & 103 \\
BNIP3 & CTGAAACAGATACCCATAGCATT & CCGACTTGACCAATCCCA & 131 \\
\hline GABARAPL1 & GGTCCCCGTGATTGTAGAGA & GGAGGGATGGTGTTGTTGAC & 174 \\
\hline
\end{tabular}

The autophagy inhibitor 3-MA was added to myotubes after 4 days of differentiation at a concentration of $500 \mu \mathrm{M}$ for $18 \mathrm{hr}$. These conditions were chosen because they can induce a significant decrease in autophagy marker expression associated with an increase in the COPD myotube diameter, while higher 3-MA concentrations do not improve these effects (data not shown).

Ascorbic acid was added at a concentration of $0.14 \mathrm{mM}$ to the culture medium of myoblasts, 1 day before induction of differentiation. Ascorbic acid was then renewed every day until the end of the differentiation, as previously described (Pomiès et al., 2016).

\subsection{Fluorescence microscopy and image analysis}

Myoblasts or myotubes were fixed for $15 \mathrm{~min}$ in $\mathrm{PBS} / 3.7 \%$ formaldehyde, permeabilized in PBS/0.1\% Triton X-100 for 5 min,
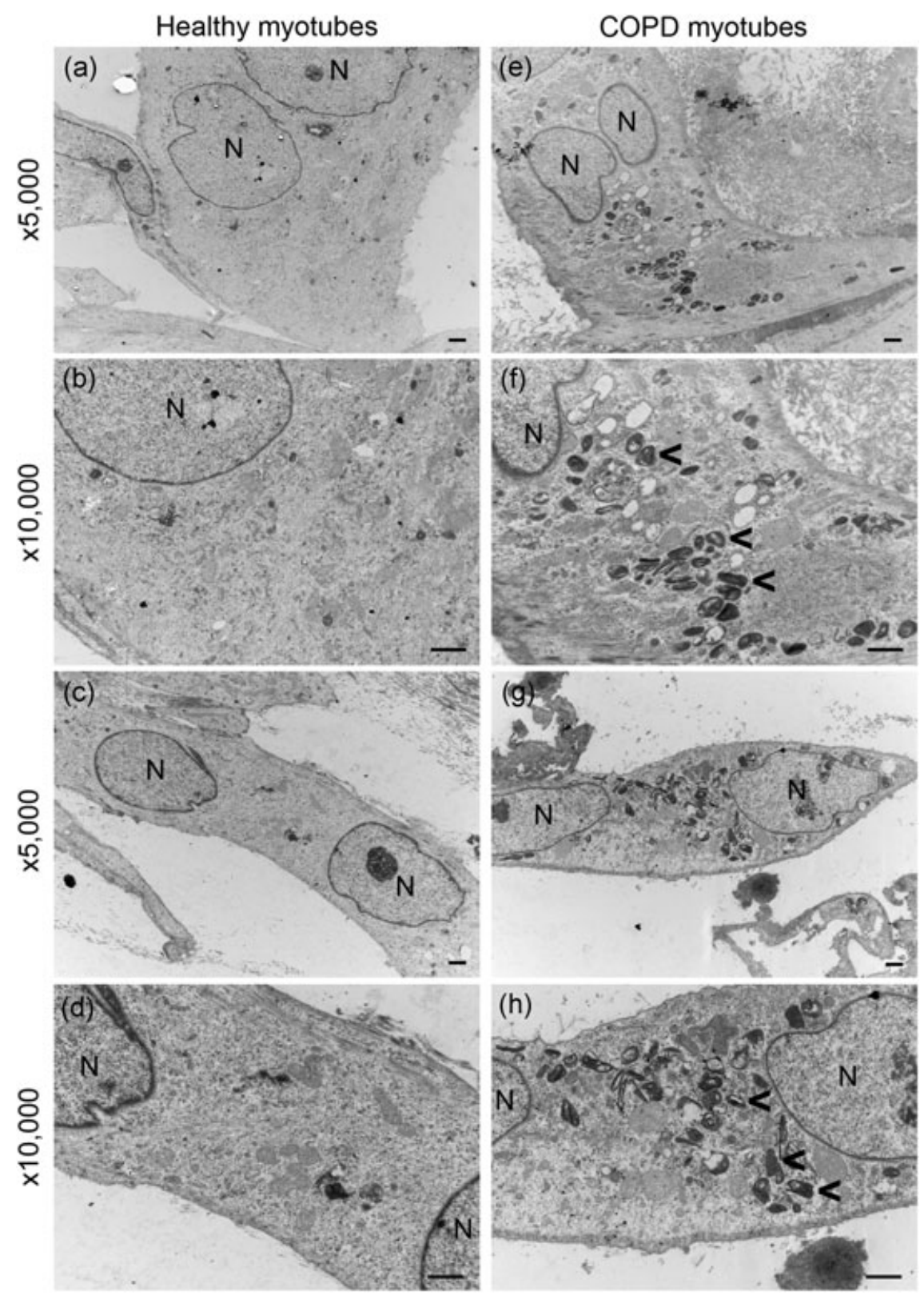

FIGURE 1 Autophagosomes in healthy and COPD myotubes. Representative electron micrographs of healthy subject myotubes $(a-d)$ and COPD patient myotubes (e-h). b, d, $f$ and $h$ are blow-up images $(\times 10,000)$ of a, c, e and $g$ images $(x 5,000)$, respectively. Arrows point towards autophagosomes, while $\mathrm{N}$ indicates a nucleus. $\mathrm{Bar}=1 \mu \mathrm{m}$ 
and then stained for $1 \mathrm{hr}$ at $37^{\circ} \mathrm{C}$ with an anti-LC3 antibody and Hoechst 33258. Images were taken in five random fields using an AxioCam MRm CCD camera (Carl Zeiss, Oberkochen, Germany) on an Axiolmager M1 microscope (Carl Zeiss) at 10x magnification. Images were anonymized and the LC3 staining density for each image was analyzed using ImageJ (National Institutes of Health, Bethesda, MD), as previously described (Zhang et al., 2014). For assessment of the myotube diameter, myotubes were first stained with an antitroponin T antibody and Hoechst 33258. Images were captured in five random fields at $5 \times$ magnification and anonymized. The diameter was then assessed by measuring three diameters along each myotube, for 100 myotubes per condition.

\section{5 | Electron microscopy}

Myotubes were immersed in a solution of $2.5 \%$ glutaraldehyde in PHEM (PIPES, HEPES, EGTA and $\mathrm{MgCl}_{2}$ ) buffer $\mathrm{pH} 7.4$ overnight at $4^{\circ} \mathrm{C}$. They were then rinsed in PHEM buffer and fixed in $0.5 \%$ osmic acid for $2 \mathrm{hr}$ in the dark at room temperature. After two rinses in PHEM buffer, the cells were dehydrated in a graded series of ethanol solutions 30\%-100\%). The cells were then embedded in EMbed 812 (Electron Microscopy Sciences, Hatfield, PA) using an EM AMW automatic microwave tissue processor (Leica Microsystems, Wetzlar, Germany). Thin sections of $70 \mathrm{~nm}$ were collected with an Ultracut $\mathrm{E}$ microtome (Leica) at different levels of each block. These sections were counterstained with $1.5 \%$ uranyl acetate in $70 \%$ ethanol and lead citrate and observed using a H7100 transmission electron microscope (Hitachi, Tokyo, Japan).

\subsection{SDS-PAGE and immunoblotting}

Proteins were extracted from myoblasts and myotubes with hypotonic buffer: $20 \mathrm{mM}$ Tris $\mathrm{pH} 7.5 / 10 \mathrm{mM} \mathrm{NaCl} / 1 \mathrm{mM}$ DTT/ protease inhibitor cocktail, separated by SDS-PAGE and transferred to Immobilon-P PVDF membranes (Millipore, Bedford, MA). LC3 1 and LC3 2 were detected with a specific anti-LC3A/B antibody. The membranes were analyzed on an Odyssey imaging system (LI-COR Biotechnology, Lincoln, NE).

\section{7 | Quantitative polymerase chain reaction}

Total RNA extraction from cultured cells, first strand complementary DNA synthesis, and quantitative polymerase chain reaction protocol and analysis have been described (Pomiès et al., 2016). Specific values were normalized to the values of the housekeeping gene GAPDH. The primer sequences are shown in Table 2 .

\section{8 | Reagents and antibodies}

Mouse monoclonal anti-troponin $\mathrm{T}$ was purchased from SigmaAldrich (Saint Louis, MO), and rabbit polyclonal anti-LC3A/B and rabbit monoclonal anti-SQSTM1/p62 from Cell Signaling Technology
(Danvers, MA). Hoechst 33258, 3-MA, bafilomycin A1 and ascorbic acid were purchased from Sigma-Aldrich.

\section{9 | Statistical analysis}

The variables of the healthy control and COPD patient groups were compared with the Student t-test. The percentage of variation between the "with" and "without" treatment was compared with a reference value of 100 using the Student t-test. Spearman's coefficient was used to assess correlations between variables. The variations in the myotube diameter of the study groups "with" or "without" 3-MA treatment were compared using the Wilcoxon signed rank test. Statistical analysis was performed using GraphPad Prism 5 (GraphPad Software, San Diego, CA).

\section{3 | RESULTS}

\subsection{Characteristics of the study groups}

Table 1 illustrates the main clinical and functional characteristics of the healthy subjects $(n=8)$ and patients with COPD $(n=12)$. The following values reflected the moderate to severe clinical state of the COPD group: forced expiratory volume in 1 s (FEV1) lower than $50 \%$ predicted, moderately reduced 6-min walking distance (6MWD) and quadriceps muscle voluntary contraction (qMVC) values, and a relatively high BODE index (Celli et al., 2004).

\subsection{Elevated autophagy signaling in COPD myoblasts and myotubes}

To evaluate the autophagosome formation level in the cultured COPD myotubes, autophagosomes were detected using transmission electron microscopy. Representative images of myotube cultures from two healthy subjects and two patients with COPD showed the abundance of autophagosomes in COPD myotubes compared with control myotubes (Figure 1). To quantify autophagosome formation, the expression level of the autophagosome marker, LC3, was assessed using immunofluorescence microscopy in myoblasts and myotubes derived from a limited number of patients with COPD and healthy subjects from our study groups. Representative images of one control and one COPD myoblast culture, as well as one control and one COPD myotube culture, are presented in Figure 2a,b,d,e, respectively. Figure 2b,e showed typical dot-like staining of autophagosomes in COPD myoblasts and myotubes, respectively. Analysis of the LC3 expression level showed significantly increased autophagosome formation in COPD myoblasts ( $p=0.011$; Figure $2 c$ ) and COPD myotubes ( $p<0.001$; Figure 2f) compared with healthy subject cells.

To confirm the elevated autophagy signaling in COPD myoblasts and myotubes, several autophagy-related markers were assessed. The LC3 2/LC3 1 ratio was significantly higher in COPD myoblasts compared with muscle cells from healthy subjects 
$(p=0.002$; Figure 3a). In addition, SQSTM1 mRNA and protein as well as BNIP3 mRNA expression in COPD myoblasts (Figure 3b,c) was significantly more elevated than in myoblasts from healthy subjects ( $p=0.023, p=0.007, p=0.031$, respectively). No significant difference in GABARAPL1 expression was detected in the COPD muscle cells (Figure $3 d$ ).

To determine whether the enhanced expression of the autophagic markers in COPD myoblasts was due to an enhanced induction of autophagy or a reduced autophagic degradation, the autophagic flux was assessed using the lysosomal inhibitor bafilomycin A1. The LC3 2 autophagic flux was significantly more elevated in COPD myoblasts than in healthy myoblasts ( $p=0.002$; Figure $4 a$ ), whereas no difference was observed between COPD and healthy myotubes (Figure 4b).

To assess the link between the enhanced autophagy signaling we observed in vitro and the increased autophagy described in the lower limb muscles of patients with COPD by others (Guo et al., 2013; Puig-Vilanova et al., 2015), we determined the expression of the autophagy marker BNIP3 in quadriceps biopsies obtained from five healthy subjects and six patients with COPD of our study groups. Although the BNIP3 expression in the patients with COPD was not significantly higher than that in the healthy subjects ( $p=$ 0.219; Figure 5a), BNIP3 expression in quadriceps biopsies was positively correlated with the BNIP3 expression in the cultured myoblasts $(r=0.663 ; p=0.031$; Figure 5b). Interestingly, the BNIP3 expression in muscle biopsies was inversely correlated with both FEV1 (\% predicted) ( $r=-0.645 ; p=0.037$; Figure $5 c$ ) and 6MWD (\% predicted) ( $r=-0.736 ; p=0.013$; Figure $5 d$ ) of the study subjects. A statistical significance at $p<0.05$ is still observed for the three presented correlations when the outer COPD value is removed.
FIGURE 2 LC3 expression levels in healthy and COPD myoblasts and myotubes. (a) Representative images of control healthy subject (CONT) and COPD (COPD) myoblasts, showing fluorescence double-labeling using an anti-LC3 antibody (red) and Hoechst (blue). Bar $=200 \mu \mathrm{m}$. (b) Higher magnification showing dot-like staining of autophagosomes in COPD myoblasts. Bar $=50 \mu \mathrm{m}$. (c) Densitometric analysis of the LC3 staining (arbitrary unit) in myoblasts from five healthy subjects and five patients with COPD.

(d) Representative images of control healthy subject (CONT) and COPD (COPD) myotubes, showing fluorescence double-labeling using an anti-LC3 antibody (red) and Hoechst (blue). Bar $=200 \mu \mathrm{m}$. (e) Higher magnification showing dot-like staining of autophagosomes in COPD myotubes. Bar $=50 \mu \mathrm{m}$. (f) Densitometric analysis of the LC3 staining (arbitrary unit) in myotubes from five healthy subjects and five patients with COPD. $\left({ }^{*}\right)$ and $\left({ }^{* * *}\right)$ indicate statistical significance at $p<0.05$ and $p<0.001$, respectively. The medians are indicated. COPD, chronic obstructive pulmonary disease [Color figure can be viewed at wileyonlinelibrary.com] (a)

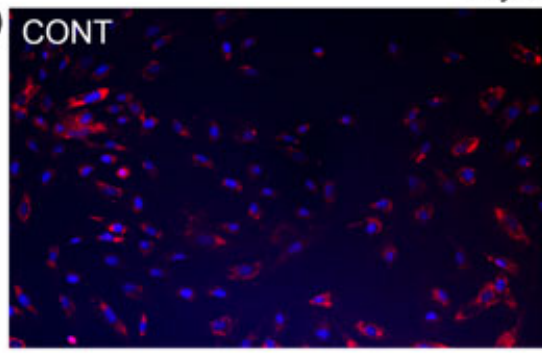

Myoblasts

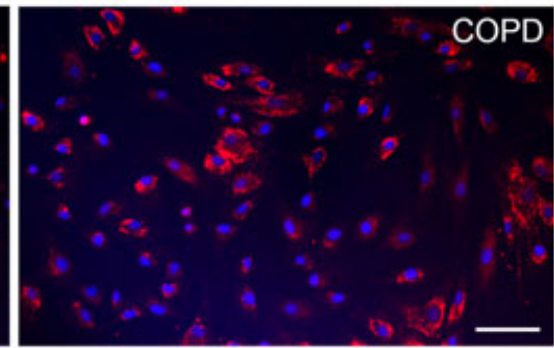

(b)
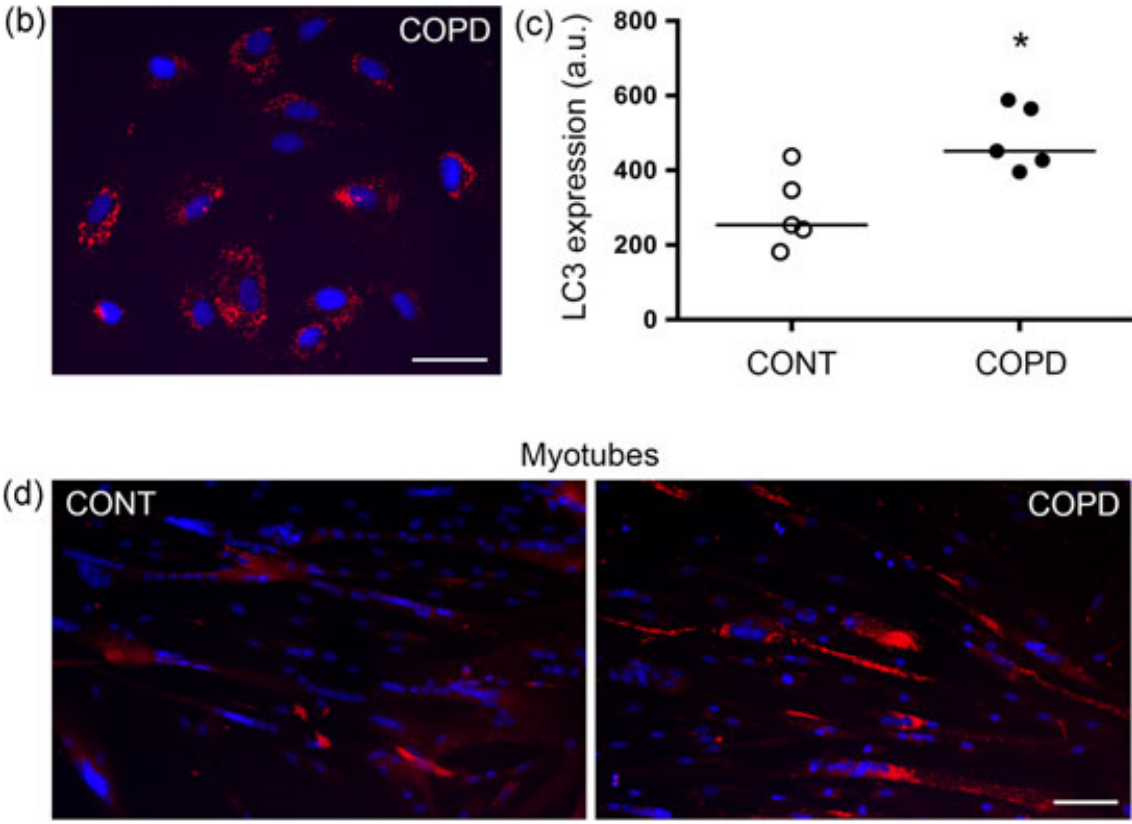

Myotubes (e)

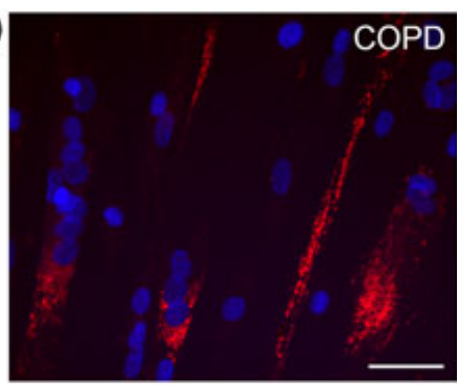

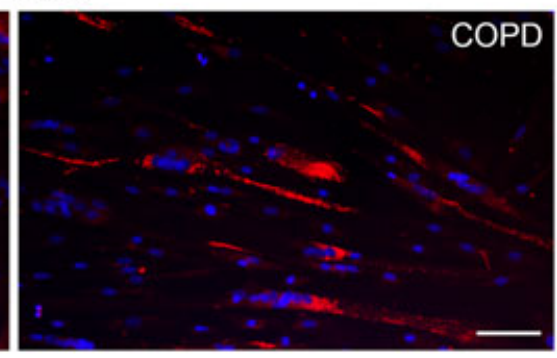

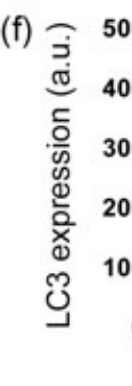

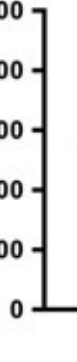

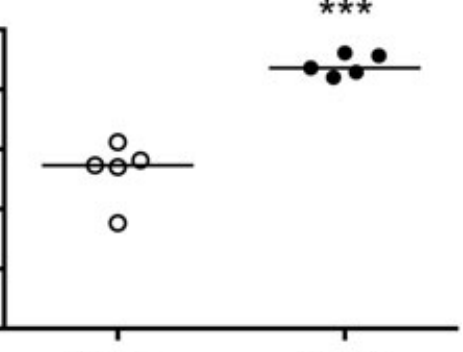

CONT 
(a)

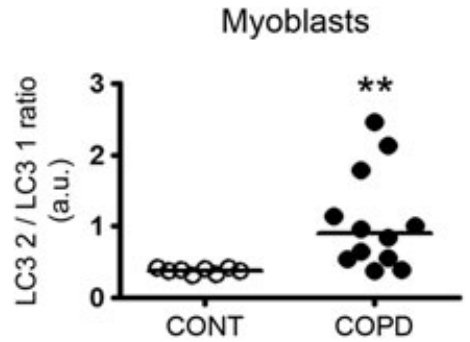

(b)

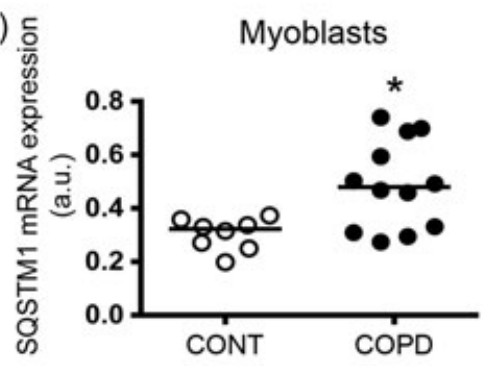

(c)

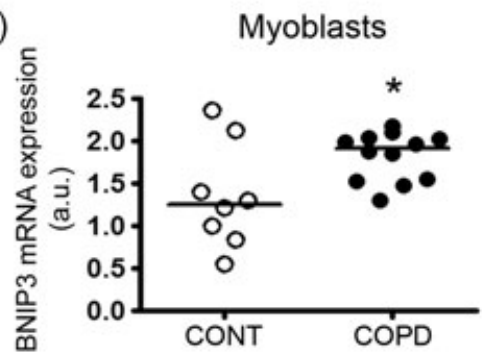

(d)

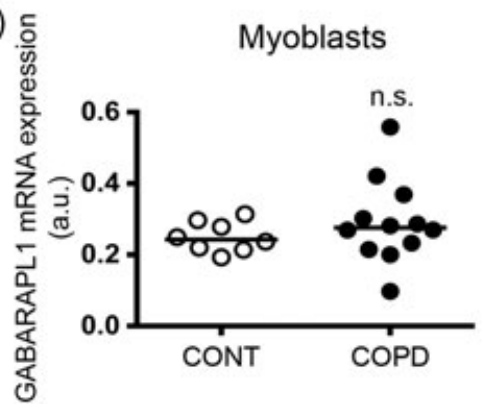

Myotubes
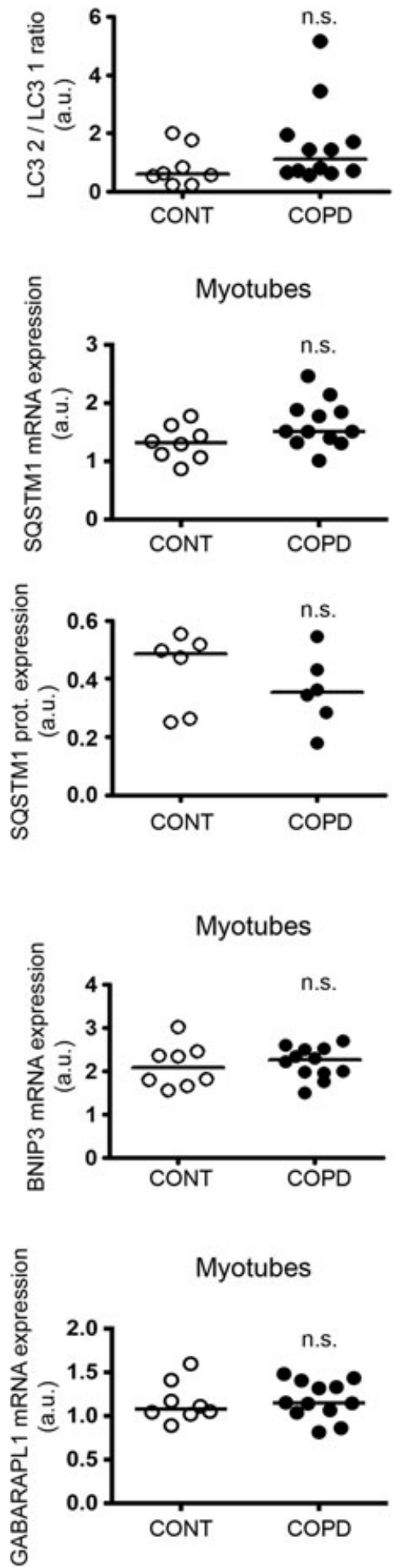

FIGURE 3 Expression of autophagyrelated markers in healthy and COPD myoblasts and myotubes. (a) Analysis of the LC3 2/LC3 1 ratio, from the quantification of two independent western blots, in eight control healthy (CONT) and twelve COPD (COPD) myoblasts and myotubes. The mean values are presented in arbitrary units. Expression levels (arbitrary unit) of SQSTM1 mRNA and protein (b), BNIP3 mRNA (c) and GABARAPL1 mRNA (d), in myoblasts and myotubes derived from healthy subjects and patients with COPD. $\left({ }^{*}\right)$ and $\left({ }^{* *}\right)$ indicate statistical significance at $p<0.05$ and $p<0.01$, respectively. (n.s.) indicates statistically nonsignificant. The medians are indicated. COPD, chronic obstructive pulmonary disease

\subsection{Atrophy is reduced in 3-MA-treated COPD myotubes}

To investigate the involvement of autophagy in the atrophy of the cultured COPD myotubes, the autophagy inhibitor 3-MA was added to the culture medium of the myotubes derived from twelve patients with COPD. Treatment induced the inhibition of autophagy signaling as depicted by the significant decrease in SQSTM1 and BNIP3 ( $p=0.001, p=0.017$, respectively; Figure $6 \mathrm{a}$ ), and a tendency to a decreased expression of GABARAPL1 ( $p=0.060$; Figure 6a). Representative images of COPD myotubes cultured in the absence and the presence of the autophagy inhibitor are shown in Figure 6b. Analysis of the COPD cultures indicated that 3-MA significantly increased the myotube diameter ( $p<0.001$; Figure 6b). However, 3-MA treatment 
(a)

\section{Myoblasts}

CONT COPD

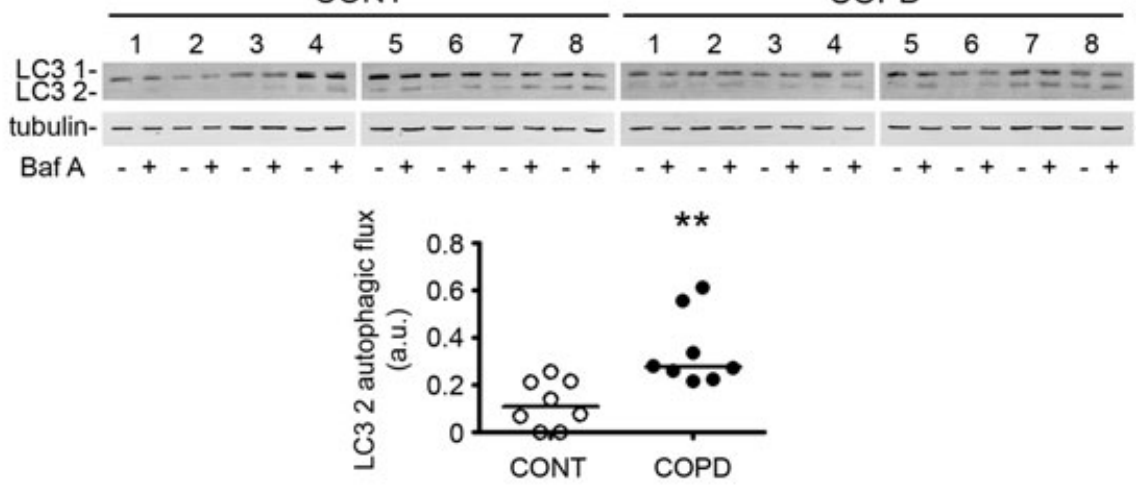

(b)

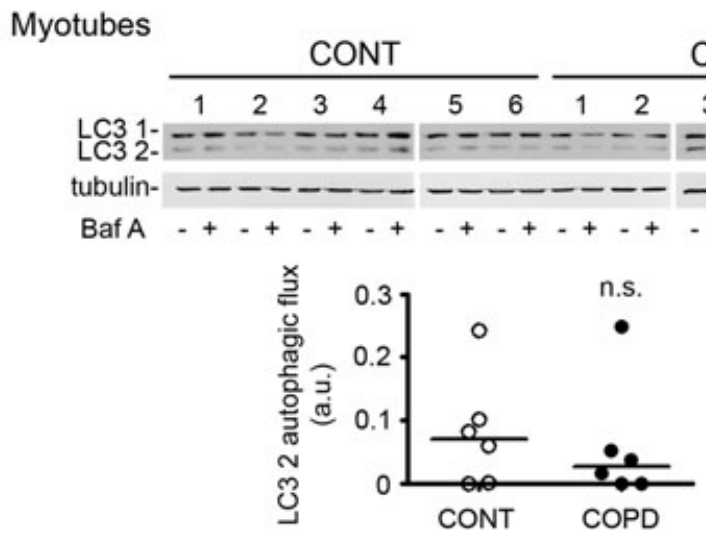

FIGURE 4 LC3 2 autophagic flux in healthy and COPD myoblasts and myotubes. (a) Representative western blots showing the expression levels of LC3 1, LC3 2 and tubulin in cultured myoblasts derived from eight control healthy subjects (CONT 1-8) and eight patients with COPD (COPD 1-8) in absence or in presence of bafilomycin A1, and analysis of the LC3 2 autophagic flux in the eight control healthy (CONT) and eight COPD (COPD) myoblasts. (b) Representative western blots showing the expression levels of LC3 1, LC3 2 and tubulin in cultured myotubes derived from six control healthy subjects (CONT 1-6) and six patients with COPD (COPD 1-6) in absence or in presence of bafilomycin A1, and analysis of the LC3 2 autophagic flux in the six control healthy (CONT) and six COPD (COPD) myoblasts. $\left({ }^{* *}\right)$ indicates statistical significance at $p<0.01$. (n.s.) indicates statistically nonsignificant. COPD, chronic obstructive pulmonary disease

of cultured myotubes derived from healthy subjects did not increase the myotube diameter ( $p=0.641$; Figure $6 c$ ). Interestingly, treatment of the COPD myotubes with the autophagy inhibitor 3-MA could increase their diameter to a level similar to the diameter of healthy myotubes (Figure 6b,c, lower panels).

\subsection{Atrophy is decreased in acid ascorbic-treated COPD myotubes}

We previously reported that antioxidant treatment of COPD muscle cells with ascorbic acid significantly decreased ROS concentration and oxidative stress-induced cellular damage, and increased myotube diameter (Pomiès et al., 2016) ( $p<0.001, p=0.019, p<0.001$, respectively; Figure 7a). To determine whether oxidative stress is also involved in the elevated autophagy signaling of COPD muscle cells, we assessed the effect of ascorbic acid treatment of COPD muscle cells on the expression of autophagy-related markers. As shown in Figure $7 \mathrm{~b}$, the antioxidant treatment induced a significant decrease in the LC3 2/LC3 1 ratio and the SQSTM1 and BNIP3 expression levels ( $p=0.037, p<0.001, p<0.001$, respectively). No variation was observed for GABARAPL1 expression $(p=0.998$; Figure $7 b)$.

\section{4 | DISCUSSION}

Although oxidative stress has been shown to regulate autophagy signaling in skeletal muscles, its involvement in the skeletal muscle autophagy of patients with COPD has never been demonstrated. In the current study, we used a cellular model to show that autophagosome formation was increased in COPD myoblasts and myotubes compared with healthy control cells (Figures 1 and 2), along with increased expression of autophagy markers and enhanced autophagic flux in COPD myoblasts (Figures 3 and 4). We also showed that in vitro autophagy signaling was correlated with the autophagy signaling observed in the quadriceps of the study subjects (Figure 5). Use of the 3-MA autophagy inhibitor confirmed the involvement of the autophagy pathway in the atrophy of cultured COPD myotubes (Figure 6). Most interestingly, the use of an antioxidant molecule on COPD myotubes, which resulted in a 
(a)

(c)

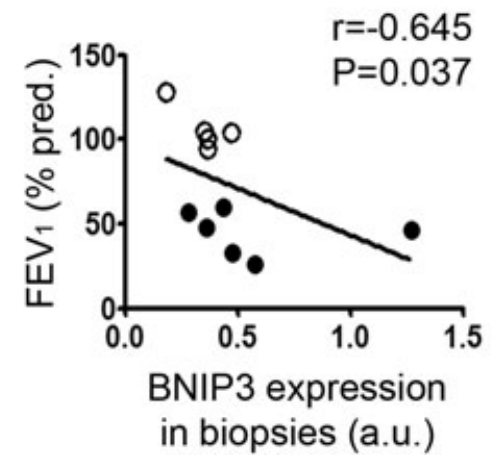

(b)

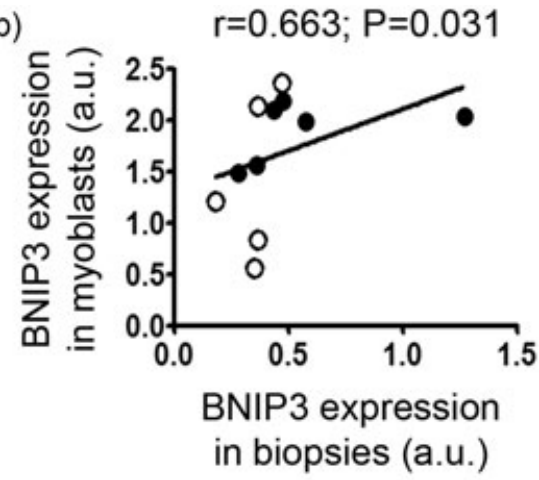

(d)

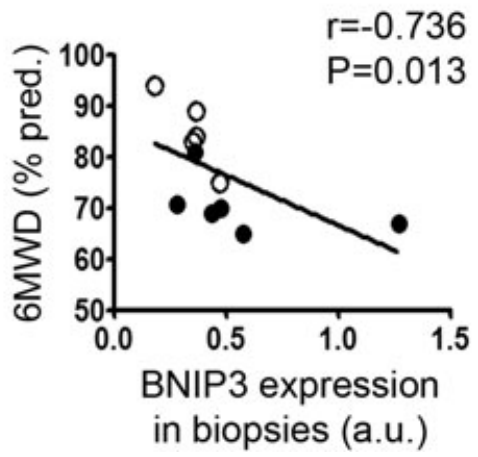

FIGURE 5 BNIP3 expression levels in the quadriceps of healthy subjects and patients with COPD. (a) Expression levels of BNIP3 in the quadriceps biopsies obtained from five healthy subjects and six patients with COPD of our study groups. (n.s.) indicates statistically nonsignificant. The medians are indicated. (b) Statistical analysis showing the correlation between BNIP3 quadriceps expression and BNIP3 expression in cultured myoblasts derived from the same healthy subjects and patients with COPD. Statistical analysis showing correlations between BNIP expression in the quadriceps and (c) FEV1 (\% predicted) and (d) 6MWD (\% predicted) of the healthy subjects and patients with COPD. Healthy subjects: open circles; patients with COPD: filled circles. COPD, chronic obstructive pulmonary disease; FEV1, forced expiratory volume in $1 \mathrm{~s} ; 6 \mathrm{MWD}$, 6-min walking distance

decrease of ROS concentration and an increase in myotube diameter, induced a significant decrease in autophagy signaling (Figure 7). Taken together, our findings showed that autophagy signaling was increased in cultured COPD muscle cells, where it played a role in the atrophy of COPD myotubes in vitro, and that oxidative stress was involved in COPD muscle autophagy.

Muscle dysfunction and atrophy are common processes that have been associated with a decreased survival rate in patients with COPD independent of the pulmonary obstruction (Marquis et al., 2002). Atrophy of the lower limb muscles of patients has been shown to result mostly from elevated protein degradation due to increased ubiquitin/proteasome signaling (Doucet et al., 2007; Plant et al., 2010). Recently, autophagosome formation and autophagy marker expression were shown to be increased in the quadriceps of both, patients with COPD with mild to severe airflow obstruction (Guo et al., 2013) and patients with cachectic COPD (Puig-Vilanova et al., 2015), compared with healthy subjects. Furthermore, autophagosome formation was inversely correlated with FEV1 (\% predicted), whereas the LC3 2/LC3 1 ratio was negatively correlated with the thigh cross-sectional area, suggesting a link between autophagyassociated muscle degradation and COPD muscle atrophy, with a protective role of autophagy toward muscle function (Guo et al., 2013). Our in vitro studies showed that autophagosome formation, autophagy-related marker expression, and autophagic flux were enhanced in COPD myoblasts and/or myotubes compared with muscle cells derived from sedentary healthy subjects (Figures 1-4). However, since SQSTM1 is an adapter protein of the autophagic degradation process, one could expect a decrease in expression of SQSTM1 in COPD muscle cells. Nevertheless, our result showing increased expression of SQSTM1 in COPD myoblasts (Figure 3) is in agreement with the enhanced expression of mRNA and protein SQSTM1 observed in the vastus lateralis of patients with COPD (Guo et al., 2013). Furthermore, it has been shown that an enhanced expression of SQSTM1 can occur in parallel with activated autophagic flux in some situations, including autophagy activation in cardiac proteinopathy (Zheng, Su, Ranek, \& Wang, 2011) and autophagy induction, contributing to muscle wasting in mouse models of cancer cachexia (Penna et al., 2013). It is, therefore, reasonable to think that in COPD muscle cells, an increased expression of SQSTM1 is associated with an enhanced autophagic flux.

Unlike a previous report (Guo et al., 2013), we did not detect a significant difference in the quadriceps expression of BNIP3 in healthy subjects and patients with COPD, but this discrepancy might be due to the relatively low number of quadriceps biopsies available for our study. In their study, Guo et al. (2013) found an inverse 
(a)

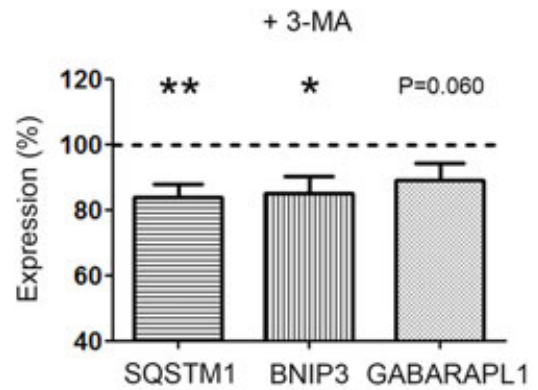

(b)

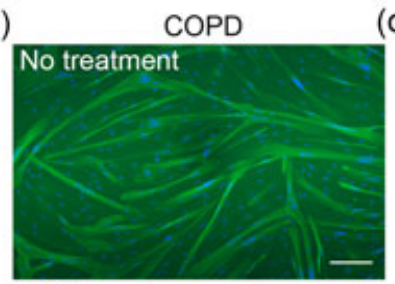

(c)
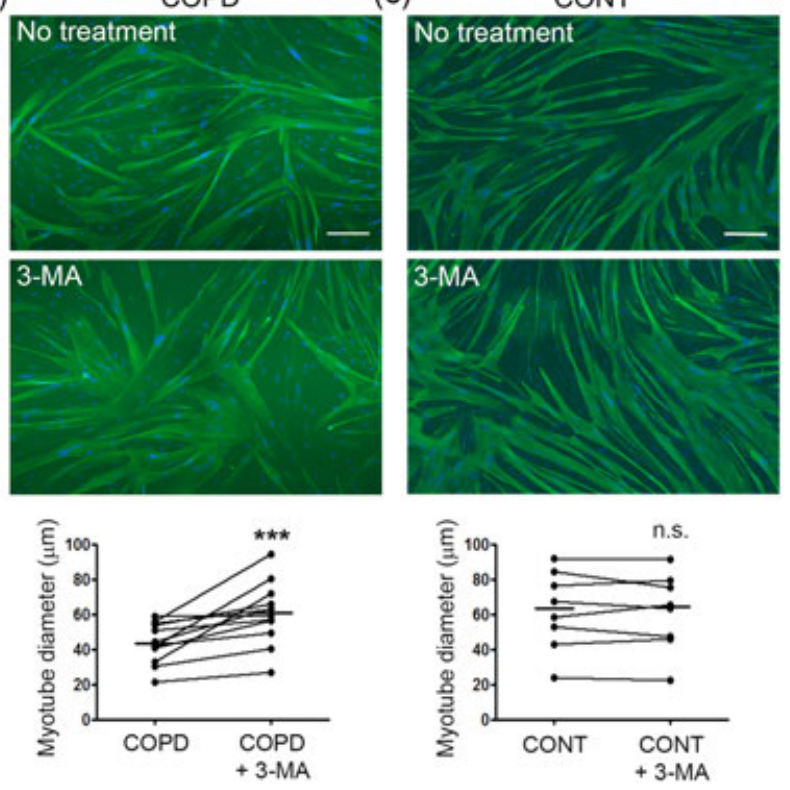

FIGURE 6 Expression levels of autophagy-related markers and myotube diameter after 3-MA treatment of COPD and healthy myotubes. (a) SQSTM1, BNIP3, and GABARAPL1 expression levels in myotubes derived from twelve patients with COPD with 3-MA treatment, relative to the expression levels of the same markers without treatment (dotted line). The means \pm SEM are indicated. (b) Representative images of COPD myotubes without or with 3-MA treatment, and analysis of the variation of the diameter of myotubes derived from twelve patients with COPD after 3-MA treatment.

(c) Representative images of healthy myotubes without or with 3-MA treatment, and analysis of the variation of the diameter of myotubes derived from eight healthy subjects after 3-MA treatment. The medians are indicated. $\left({ }^{*}\right),\left({ }^{* *}\right)$ and $\left({ }^{* * *}\right)$ indicate statistical significance at $p<0.05$, $p<0.01$ and $p<0.001$, respectively. (n.s.) indicates statistically nonsignificant. Bar $=200 \mu \mathrm{m}$. COPD, chronic obstructive pulmonary disease; 3-MA, 3-methyladenine; SEM, standard error of the mean [Color figure can be viewed at wileyonlinelibrary.com]

correlation between markers of autophagosome formation (autophagosomes/field, LC3 intensity, LC3 2/LC3 1 ratio) in the lower limb muscles and lung and muscle function (FEV1 [\% predicted], FEV1/ forced vital capacity [\%], thigh cross-sectional area). In our study, we observed similar significant correlations between autophagy signaling (BNIP3 expression) in the quadriceps and FEV1 (\% predicted) and 6MWD (\% predicted; Figure 5), although our experimental study would have benefited from a larger population of patients with COPD with various severities. We also observed a (a)

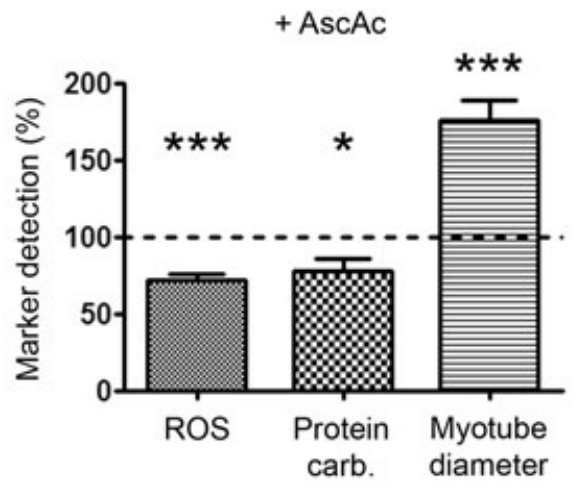

(b)

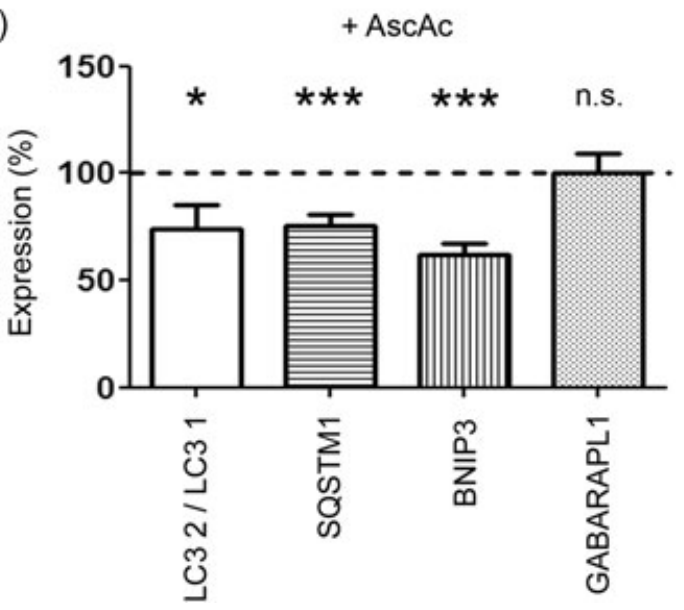

FIGURE 7 Expression levels of autophagy-related markers in COPD myotubes after ascorbic acid treatment. (a) Analysis of ROS concentration, protein carbonylation level and myotube diameter in cultured myotubes derived from twelve patients with COPD after ascorbic acid treatment, relative to the detection of the same markers without treatment (dotted line). (b) Analysis of the LC3 2/LC3 1 ratio, and SQSTM1, BNIP3 and GABARAPL1 expression levels in cultured myotubes derived from twelve patients with COPD with ascorbic acid treatment, relative to the expression levels of the same markers without treatment (dotted line). $\left({ }^{*}\right)$ and $\left({ }^{* * *}\right)$ indicate statistical significance at $p<0.05$ and $p<0.001$, respectively. (n.s.) indicates statistically nonsignificant. The means \pm SEM are indicated. COPD, chronic obstructive pulmonary disease; ROS, reactive oxygen species; SEM, standard error of the mean

correlation between BNIP3 expression in the quadriceps of the study subjects and BNIP3 expression in the cultured myoblasts (Figure 5). Together, these observations indicate that the increased autophagy signaling we identified in the cultured COPD muscle cells derived from human satellite cells reflects the enhanced autophagy observed in the quadriceps muscle of the stable patients with COPD (Guo et al., 2013; Puig-Vilanova et al., 2015).

Autophagy, a major catabolic process in the elimination of damaged molecules and organelles, plays a crucial role in muscle cell homeostasis. A relationship between autophagy and atrophy has been demonstrated in skeletal muscles subjected to various atrophic stimuli such as starvation and denervation (Mizushima et al., 2004; O'Leary et al., 2012). In the skeletal muscles of patients with COPD, it was proposed that enhanced autophagy participates in the 
remodeling of the muscle, contributing to muscle atrophy (Hussain \& Sandri, 2013). In our study, treatment of atrophic COPD myotubes with the autophagy inhibitor 3-MA induced a drastic increase in the myotube diameter, showing that autophagy was involved in the atrophy of COPD muscle cells in vitro (Figure 6).

Although the pro-oxidant molecule, hydrogen peroxide, has been shown to activate autophagy in cultured C2C12 myotubes (McClung, Judge, Powers, \& Yan, 2010; Rahman et al., 2014), administration of the antioxidant molecule NAC to mice reduced the autophagy signaling (Qi et al., 2014; Rahman et al., 2014), suggesting that oxidative stress regulates skeletal muscle autophagy. Direct evidence of the involvement of oxidative stress in skeletal muscle autophagy was reported in a study using a transgenic mouse model expressing a mutant of the antioxidant enzyme superoxide dismutase 1 selectively in skeletal muscle (Dobrowolny et al., 2008). Nevertheless, the involvement of oxidative stress in COPD muscle autophagy has never been studied. Here, we showed that antioxidant treatment of COPD myotubes with ascorbic acid significantly decreased ROS concentration, oxidative stress-induced cellular damage, as well as expression of various autophagy markers, and increased the myotube diameter (Figure 7). Therefore, lowering the oxidative burden in COPD muscle cells reduced the autophagy signaling, showing for the first time that the oxidative stress level regulates autophagy in COPD skeletal muscle cells. This result suggests that reduction of autophagy by antioxidant molecules might be a potential therapeutic strategy to counteract oxidative stress-induced atrophy in patients with COPD.

Mitophagy is an autophagic process dedicated to the selective elimination of damaged and dysfunctional mitochondria to avoid excessive ROS generation and preserve muscle homeostasis (Youle \& Narendra, 2011). In COPD skeletal muscles, it was shown that the altered functioning of mitochondria led to enhanced mitochondrial ROS production linked to skeletal muscle oxidative stress (PuenteMaestu et al., 2009, 2012). It would, therefore, be of interest to determine whether the increased ROS concentration we have observed in cultured COPD myoblasts and myotubes is coming from altered mitochondria. Furthermore, our results showed that the expression levels of SQSTM1 and BNIP3, two molecular adapters targeting mitochondria to autophagosomes for mitophagy, were increased in cultured COPD muscle cells compared with healthy control cells (Figure 3), and that ascorbic acid reduced their expression (Figure 7). These results suggest that oxidative stress is involved in the autophagy-associated remodeling of the mitochondrial network by the removal of dysfunctional mitochondria in the atrophic COPD muscle cells, a process that will be addressed in a forthcoming study.

In summary, we assessed autophagosome formation, autophagy marker expression, and autophagic flux and showed that autophagy signaling was enhanced in cultured muscle cells derived from patients with COPD compared with those from healthy subjects, reflecting the increased autophagy signaling observed in the quadriceps of patients with COPD. Furthermore, treatment of COPD myotubes with an autophagy inhibitor significantly decreased their atrophic phenotype, confirming the involvement of autophagy in the atrophy of COPD muscle cells. Last, antioxidant treatment diminished the ROS concentration and the autophagy signaling and increased the COPD myotube diameter. These data are therefore the first evidence that the oxidative stress level contributes to the regulation of autophagy in COPD muscle cells and that autophagy may be involved in the atrophy of COPD skeletal muscles. While little information is available on how oxidative stress levels affect autophagy in muscle, such in vitro approaches should help us to decipher this signaling pathway in COPD muscles.

\section{ACKNOWLEDGMENTS}

We thank Nicolas Molinari (IMAG, Montpellier, France) for his advice on statistical analysis, and the Fontalvie/5-Santé Group for the use of their facilities during the clinical evaluation and biopsies of the patients with COPD. We also acknowledge Chantal Cazevielle for her expertize with electron microscopy (Centre de Ressources en Imagerie Cellulaire de Montpellier, France).

\section{CONFLICTS OF INTEREST}

The authors declare that they have no conflict of interests.

\section{ORCID}

Pascal Pomiès (D) http://orcid.org/0000-0002-5301-6087

\section{REFERENCES}

Bellot, G., Garcia-Medina, R., Gounon, P., Chiche, J., Roux, D., Pouyssegur, J., \& Mazure, N. M. (2009). Hypoxia-induced autophagy is mediated through hypoxia-inducible factor induction of BNIP3 and BNIP3L via their BH3 domains. Molecular and Cellular Biology, 29, 2570-2581.

Celli, B. R., Cote, C. G., Marin, J. M., Casanova, C., Montes de Oca, M., Mendez, R. A., ... Cabral, H. J. (2004). The body-mass index, airflow obstruction, dyspnea, and exercise capacity index in chronic obstructive pulmonary disease. The New England Journal of Medicine, 350, 1005-1012.

Dobrowolny, G., Aucello, M., Rizzuto, E., Beccafico, S., Mammucari, C., Bonconpagni, S., ... Musarò, A. (2008). Skeletal muscle is a primary target of SOD1G93A-mediated toxicity. Cell Metabolism, 8, 425-436.

Doucet, M., Russell, A. P., Léger, B., Debigaré, R., Joanisse, D. R., Caron, M. -A., ... Maltais, F. (2007). Muscle atrophy and hypertrophy signaling in patients with chronic obstructive pulmonary disease. American Journal of Respiratory and Critical Care Medicine, 176, 261-269.

Gouzi, F., Abdellaoui, A., Molinari, N., Pinot, E., Ayoub, B., LaoudjChenivesse, D., ... Préfaut, C. (2013). Fiber atrophy, oxidative stress, and oxidative fiber reduction are the attributes of different phenotypes in chronic obstructive pulmonary disease patients. Journal of Applied Physiology, 115, 1796-1805.

Guo, Y., Gosker, H. R., Schols, A. M. W. J., Kapchinsky, S., Bourbeau, J., Sandri, M., ... Hussain, S. N. A. (2013). Autophagy in locomotor muscles of patients with chronic obstructive pulmonary disease. American Journal of Respiratory and Critical Care Medicine, 188, 1313-1320.

Hussain, S. N. A., \& Sandri, M. (2013). Role of autophagy in COPD skeletal muscle dysfunction. Journal of Applied Physiology, 114, 1273-1281.

Marquis, K., Debigaré, R., Lacasse, Y., LeBlanc, P., Jobin, J., Carrier, G., \& Maltais, F. (2002). Midthigh muscle cross-sectional area is a better 
predictor of mortality than body mass index in patients with chronic obstructive pulmonary disease. American Journal of Respiratory and Critical Care Medicine, 166, 809-813.

McClung, J. M., Judge, A. R., Powers, S. K., \& Yan, Z. (2010). p38 MAPK links oxidative stress to autophagy-related gene expression in cachectic muscle wasting. American Journal of Physiology Cell Physiology, 298, C542-C549.

Mizushima, N., Yamamoto, A., Matsui, M., Yoshimori, T., \& Ohsumi, Y. (2004). In vivo analysis of autophagy in response to nutrient starvation using transgenic mice expressing a fluorescent autophagosome marker. Molecular Biology of the Cell, 15, 1101-1111.

O'leary, M. F. N., Vainshtein, A., Carter, H. N., Zhang, Y., \& Hood, D. A. (2012). Denervation-induced mitochondrial dysfunction and autophagy in skeletal muscle of apoptosis-deficient animals. American Journal of Physiology Cell Physiology, 303, C447-C454.

Penna, F., Costamagna, D., Pin, F., Camperi, A., Fanzani, A., Chiarpotto, E. M., ... Costelli, P. (2013). Autophagic degradation contributes to muscle wasting in cancer cachexia. The American Journal of Pathology, 182, 1367-1378.

Plant, P. J., Brooks, D., Faughnan, M., Bayley, T., Bain, J., Singer, L., ... Batt, J. (2010). Cellular markers of muscle atrophy in chronic obstructive pulmonary disease. American Journal of Respiratory Cell and Molecular Biology, 42, 461-471.

Pomiès, P., Blaquière, M., Maury, J., Mercier, J., Gouzi, F., \& Hayot, M. (2016). Involvement of the FoxO1/MuRF1/atrogin-1 signaling pathway in the oxidative stress-induced atrophy of cultured chronic obstructive pulmonary disease myotubes. PLoS One, 11, e0160092.

Pomiès, P., Rodriguez, J., Blaquière, M., Sedraoui, S., Gouzi, F., Carnac, G., ... Hayot, M. (2015). Reduced myotube diameter, atrophic signalling and elevated oxidative stress in cultured satellite cells from COPD patients. Journal of Cellular and Molecular Medicine, 19, 175-186.

Puente-Maestu, L., Perez-Parra, J., Godoy, R., Moreno, N., Tejedor, A., Gonzalez-Aragoneses, F., ... Agusti, A. (2009). Abnormal mitochondrial function in locomotor and respiratory muscles of COPD patients. The European Respiratory Journal, 33, 1045-1052.
Puente-Maestu, L., Tejedor, A., Lázaro, A., de Miguel, J., Alvarez-Sala, L., González-Aragoneses, F., \& Agustí, A. (2012). Site of mitochondrial reactive oxygen species production in skeletal muscle of chronic obstructive pulmonary disease and its relationship with exercise oxidative stress. American Journal of Respiratory Cell and Molecular Biology, 47, 358-362.

Puig-Vilanova, E., Rodriguez, D. A., Lloreta, J., Ausin, P., Pascual-Guardia, S., Broquetas, J., ... Barreiro, E. (2015). Oxidative stress, redox signaling pathways, and autophagy in cachectic muscles of male patients with advanced COPD and lung cancer. Free Radical Biology \& Medicine, 79, 91-108.

Qi, Z., He, Q., Ji, L., \& Ding, S. (2014). Antioxidant supplement inhibits skeletal muscle constitutive autophagy rather than fasting-induced autophagy in mice. Oxidative Medicine and Cellular Longevity, 2014, 315896.

Rahman, M., Mofarrahi, M., Kristof, A. S., Nkengfac, B., Harel, S., \& Hussain, S. N. (2014). Reactive oxygen species regulation of autophagy in skeletal muscles. Antioxidants \& Redox Signaling, 20, 443-459.

Youle, R. J., \& Narendra, D. P. (2011). Mechanisms of mitophagy. Nature Reviews Molecular Cell Biology, 12, 9-14.

Zhang, H., Gong, Y., Wang, Z., Jiang, L., Chen, R., Fan, X., ... Kong, X. (2014). Apelin inhibits the proliferation and migration of rat PASMCs via the activation of $\mathrm{PI} 3 \mathrm{~K} / \mathrm{Akt} / \mathrm{mTOR}$ signal and the inhibition of autophagy under hypoxia. Journal of Cellular and Molecular Medicine, 18, 542-553.

Zheng, Q., Su, H., Ranek, M. J., \& Wang, X. (2011). Autophagy and p62 in cardiac proteinopathy. Circulation Research, 109, 296-308.

How to cite this article: Gouzi F, Blaquière $M$, Catteau $M$, et al. Oxidative stress regulates autophagy in cultured muscle cells of patients with chronic obstructive pulmonary disease. J Cell Physiol. 2018;1-11. https://doi.org/10.1002/jcp.26868 